\title{
How aerosols and greenhouse gases influence the diurnal temperature range
}

Camilla W. Stjern ${ }^{1}$, Bjørn H. Samset ${ }^{1}$, Olivier Boucher ${ }^{2}$, Trond Iversen ${ }^{3}$, Jean-François Lamarque $^{4}$, Gunnar Myhre ${ }^{1}$, Drew Shindell ${ }^{5}$, Toshihiko Takemura ${ }^{6}$

${ }^{1}$ CICERO Center of International Climate Research, Oslo, Norway

${ }^{2}$ Institut Pierre-Simon Laplace, Sorbonne Université / CNRS, Paris, France

${ }^{3}$ Norwegian Meteorological Institute, Oslo, Norway

${ }^{4}$ NCAR/UCAR, Boulder, USA

${ }^{5}$ Nicholas School of the Environment, Duke University, Durham, NC, USA

${ }^{6}$ Kyushu University, Fukuoka, Japan

Corresponding author: Camilla W. Stjern, camilla.stjern@cicero.oslo.no

Tables S1-S8

Figure S1 - S5 
Table S1: Multi-model median correlations (i.e., the median of the 9 individual model correlation coefficients) between changes in DTR and a selection of variables, for the global land region.

Correlations are based on 50 yearly (the last 50 years of the 100 -year simulations) values of seasonal, regional mean changes. The table only includes coefficients for relationships that were statistically significant ( $p<0.05$ by the Student's t-test) for at least $75 \%$ of the models. Note that we also calculated correlations to surface evaporation, but as correlation coefficients were nearly identical to that of latent heat, it is not included here.

\begin{tabular}{|l|l|l|l|l|l|l|l|}
\hline & & $\begin{array}{l}\text { Cloud } \\
\text { cover }\end{array}$ & $\begin{array}{l}\text { Latent } \\
\text { heat }\end{array}$ & $\begin{array}{l}\text { Sensible } \\
\text { heat }\end{array}$ & $\begin{array}{l}\text { Clear-sky } \\
\text { downwelling } \\
\text { SW radiation }\end{array}$ & $\begin{array}{l}\text { All-sky } \\
\text { downwelling } \\
\text { SW radiation }\end{array}$ & $\begin{array}{l}\text { All-sky } \\
\text { downwelling } \\
\text { LW radiation }\end{array}$ \\
\hline \multirow{2}{*}{ C02x2 } & DJF & -0.57 & -0.39 & & & +0.58 & -0.33 \\
\cline { 2 - 8 } & JJA & -0.60 & -0.49 & +0.58 & & +0.60 & \\
\hline \multirow{2}{*}{ BCx10 } & DJF & -0.52 & -0.27 & & & +0.67 & -0.35 \\
\cline { 2 - 8 } & JJA & -0.62 & -0.46 & +0.64 & & +0.64 & \\
\hline \multirow{2}{*}{ S04x5 } & DJF & -0.50 & -0.29 & & +0.42 & +0.61 & -0.42 \\
\cline { 2 - 8 } & JJA & -0.67 & -0.55 & +0.68 & & +0.71 & \\
\hline
\end{tabular}

Table S2: Like Table S1, for the USA region.

\begin{tabular}{|l|l|l|l|l|l|l|l|}
\hline & & $\begin{array}{l}\text { Cloud } \\
\text { cover }\end{array}$ & $\begin{array}{l}\text { Latent } \\
\text { heat }\end{array}$ & $\begin{array}{l}\text { Sensible } \\
\text { heat }\end{array}$ & $\begin{array}{l}\text { Clear-sky } \\
\text { downwelling } \\
\text { SW radiation }\end{array}$ & $\begin{array}{l}\text { All-sky } \\
\text { downwelling } \\
\text { SW radiation }\end{array}$ & $\begin{array}{l}\text { All-sky } \\
\text { downwelling } \\
\text { LW radiation }\end{array}$ \\
\hline \multirow{2}{*}{ CO2x2 } & DJF & -0.64 & -0.30 & & & +0.51 & \\
\cline { 2 - 8 } & JJA & -0.74 & -0.76 & $\mathbf{+ 0 . 8 4}$ & & $\mathbf{+ 0 . 8 3}$ & \\
\hline \multirow{2}{*}{ BCx10 } & DJF & -0.67 & -0.24 & & & +0.63 & -0.30 \\
\cline { 2 - 8 } & JJA & -0.74 & -0.74 & $\mathbf{+ 0 . 8 8}$ & & $\mathbf{+ 0 . 8 6}$ & \\
\hline \multirow{2}{*}{ S04x5 } & DJF & -0.56 & & & & +0.58 & \\
\cline { 2 - 8 } & JJA & -0.78 & -0.66 & $\mathbf{+ 0 . 8 5}$ & +0.40 & +0.80 & \\
\hline
\end{tabular}

Table S3: Like Table S1, for the Europe region. Regionally averaged, DTR goes down in DJF for all drivers, consistently due to a stronger increase in $T_{\text {min }}$ than in $T_{\text {max. }}$ In JJA, DTR increases, due to a much stronger increase in $T_{\max }$ than in $\mathrm{T}_{\min }$. Cloud amounts go down in DJF and up in JJA.

\begin{tabular}{|l|l|l|l|l|l|l|l|}
\hline & & $\begin{array}{l}\text { Cloud } \\
\text { cover }\end{array}$ & $\begin{array}{l}\text { Latent } \\
\text { heat }\end{array}$ & $\begin{array}{l}\text { Sensible } \\
\text { heat }\end{array}$ & $\begin{array}{l}\text { Clear-sky } \\
\text { downwelling } \\
\text { SW radiation }\end{array}$ & $\begin{array}{l}\text { All-sky } \\
\text { downwelling } \\
\text { SW radiation }\end{array}$ & $\begin{array}{l}\text { All-sky } \\
\text { downwelling } \\
\text { LW radiation }\end{array}$ \\
\hline \multirow{2}{*}{ CO2x2 } & DJF & -0.51 & & & & +0.50 & -0.50 \\
\cline { 2 - 8 } & JJA & -0.77 & -0.58 & $\mathbf{+ 0 . 8 4}$ & & $\mathbf{+ 0 . 8 0}$ & \\
\hline \multirow{2}{*}{ BCx10 } & DJF & -0.53 & & & +0.48 & +0.57 & -0.53 \\
\cline { 2 - 8 } & JJA & -0.70 & -0.57 & +0.77 & & +0.77 & \\
\hline \multirow{2}{*}{ S04x5 } & DJF & -0.38 & & & +0.55 & +0.51 & -0.61 \\
\cline { 2 - 8 } & JJA & -0.70 & & $\mathbf{+ 0 . 8 4}$ & +0.37 & $\mathbf{+ 0 . 8 3}$ & \\
\hline
\end{tabular}


Table S4: Like Table S1, for the India region.

\begin{tabular}{|l|l|l|l|l|l|l|l|}
\hline & & $\begin{array}{l}\text { Cloud } \\
\text { cover }\end{array}$ & $\begin{array}{l}\text { Latent } \\
\text { heat }\end{array}$ & $\begin{array}{l}\text { Sensible } \\
\text { heat }\end{array}$ & $\begin{array}{l}\text { Clear-sky } \\
\text { downwelling } \\
\text { SW radiation }\end{array}$ & $\begin{array}{l}\text { All-sky } \\
\text { downwelling } \\
\text { SW radiation }\end{array}$ & $\begin{array}{l}\text { All-sky } \\
\text { downwelling } \\
\text { LW radiation }\end{array}$ \\
\hline \multirow{2}{*}{ C02x2 } & DJF & -0.61 & -0.54 & +0.67 & +0.68 & $\mathbf{+ 0 . 8 2}$ & -0.66 \\
\cline { 2 - 8 } & JJA & $-\mathbf{0 . 8 5}$ & -0.70 & $\mathbf{+ 0 . 8 7}$ & +0.59 & $\mathbf{+ 0 . 8 5}$ & -0.28 \\
\hline \multirow{2}{*}{ BCx10 } & DJF & -0.67 & -0.66 & +0.64 & +0.56 & +0.70 & -0.67 \\
\cline { 2 - 8 } & JJA & $-\mathbf{0 . 8 4}$ & -0.65 & $\mathbf{+ 0 . 8 4}$ & +0.71 & $\mathbf{+ 0 . 9 0}$ & -0.30 \\
\hline \multirow{2}{*}{ S04x5 } & DJF & -0.67 & -0.64 & $\mathbf{+ 0 . 7 5}$ & +0.64 & $\mathbf{+ 0 . 8 1}$ & -0.58 \\
\cline { 2 - 8 } & JJA & $-\mathbf{0 . 8 3}$ & -0.78 & $\mathbf{+ 0 . 8 7}$ & +0.76 & $\mathbf{+ 0 . 8 9}$ & -0.63 \\
\hline
\end{tabular}

Table S5: Like Table S1, for the China region.

\begin{tabular}{|l|l|l|l|l|l|l|l|}
\hline & & $\begin{array}{l}\text { Cloud } \\
\text { cover }\end{array}$ & $\begin{array}{l}\text { Latent } \\
\text { heat }\end{array}$ & $\begin{array}{l}\text { Sensible } \\
\text { heat }\end{array}$ & $\begin{array}{l}\text { Clear-sky } \\
\text { downwelling } \\
\text { SW radiation }\end{array}$ & $\begin{array}{l}\text { All-sky } \\
\text { downwelling } \\
\text { SW radiation }\end{array}$ & $\begin{array}{l}\text { All-sky } \\
\text { downwelling } \\
\text { LW radiation }\end{array}$ \\
\hline \multirow{2}{*}{ C02x2 } & DJF & -0.78 & & +0.43 & +0.53 & $\mathbf{+ 0 . 8 7}$ & -0.39 \\
\cline { 2 - 8 } & JJA & -0.75 & & +0.77 & & $+\mathbf{0 . 8 5}$ & \\
\hline \multirow{2}{*}{ BCx10 } & DJF & -0.73 & & +0.31 & +0.32 & +0.75 & \\
\cline { 2 - 8 } & JJA & -0.78 & & +0.70 & & $\mathbf{+ 0 . 8 6}$ & \\
\hline \multirow{2}{*}{ S04x5 } & DJF & -0.67 & & & & +0.79 & \\
\cline { 2 - 7 } & JJA & -0.75 & & +0.77 & +0.64 & $\mathbf{+ 0 . 8 1}$ & -0.41 \\
\hline
\end{tabular}

Table S6: Like Table S1, for the Arctic region, where DTR goes down for all drivers in DJF, while JJA responses are more varying (increasing DTR for $\mathrm{CO}_{2}$, due to strong increase in $\mathrm{T}_{\max }$, but reducing for $\mathrm{BC}$ and $\mathrm{SO}_{4}$ due to stronger increase in $\mathrm{T}_{\mathrm{min}}$ ).

\begin{tabular}{|l|l|l|l|l|l|l|l|}
\hline & & $\begin{array}{l}\text { Cloud } \\
\text { cover }\end{array}$ & $\begin{array}{l}\text { Latent } \\
\text { heat }\end{array}$ & $\begin{array}{l}\text { Sensible } \\
\text { heat }\end{array}$ & $\begin{array}{l}\text { Clear-sky } \\
\text { downwelling } \\
\text { SW radiation }\end{array}$ & $\begin{array}{l}\text { All-sky } \\
\text { downwelling } \\
\text { SW radiation }\end{array}$ & $\begin{array}{l}\text { All-sky } \\
\text { downwelling } \\
\text { LW radiation }\end{array}$ \\
\hline \multirow{2}{*}{ C02x2 } & DJF & & & -0.39 & & & \\
\cline { 2 - 8 } & JJA & -0.73 & +0.52 & +0.60 & & +0.77 & \\
\hline \multirow{2}{*}{ BCx10 } & DJF & & & -0.38 & & & \\
\hline & JJA & -0.76 & & +0.59 & & +0.72 & \\
\hline S04x5 & DJF & & & & & & \\
\cline { 2 - 8 } & JJA & -0.77 & & +0.58 & & +0.70 & \\
\hline
\end{tabular}

Table S7: Multi-model median cloud cover changes, normalized by global mean temperature change [\% per K].

\begin{tabular}{|l|l|l|l|l|l|l|}
\hline & \multicolumn{2}{|c|}{ CO2x2 } & \multicolumn{2}{c|}{ BCx10 } & \multicolumn{2}{c|}{ S04x5 } \\
\hline & DJF & JJA & DJF & JJA & DJF & JJA \\
\hline LND & +0.10 & -0.35 & -0.20 & -1.13 & +0.04 & -0.41 \\
\hline USA & +0.15 & -0.12 & +0.44 & -3.10 & -0.26 & -1.04 \\
\hline EUR & +0.10 & -0.75 & -0.85 & -6.60 & +0.45 & -1.18 \\
\hline IND & -0.09 & -0.0 & -0.24 & +5.65 & -0.56 & +2.16 \\
\hline CHI & +0.03 & -0.29 & +1.45 & +0.71 & -0.17 & +0.15 \\
\hline ARC & +1.04 & -0.51 & +1.27 & +0.71 & +0.88 & +0.55 \\
\hline
\end{tabular}


Table S8: Multi-model median changes in downwelling clear-sky SW radiation at the surface, normalized by global mean temperature change $[\mathrm{W} / \mathrm{m} 2$ per $\mathrm{K}]$.

\begin{tabular}{|l|l|l|l|l|l|l|}
\hline & \multicolumn{2}{|c|}{ CO2X2 } & \multicolumn{2}{c|}{ BCx10 } & \multicolumn{2}{c|}{ SO4x5 } \\
\hline & DJF & JJA & DJF & JJA & DJF & JJA \\
\hline LND & -0.97 & -1.05 & -13.64 & -20.63 & +1.34 & +2.96 \\
\hline USA & -1.18 & -0.93 & -10.15 & -20.60 & +1.82 & +5.40 \\
\hline EUR & -0.79 & -0.74 & -10.88 & -33.71 & +1.90 & +8.34 \\
\hline IND & -1.21 & -1.49 & -53.65 & -43.68 & +3.21 & +4.92 \\
\hline CHI & -1.33 & -1.16 & -46.07 & -49.48 & +3.50 & +9.23 \\
\hline ARC & -0.34 & -1.21 & -2.20 & -11.50 & +0.27 & +0.47 \\
\hline
\end{tabular}
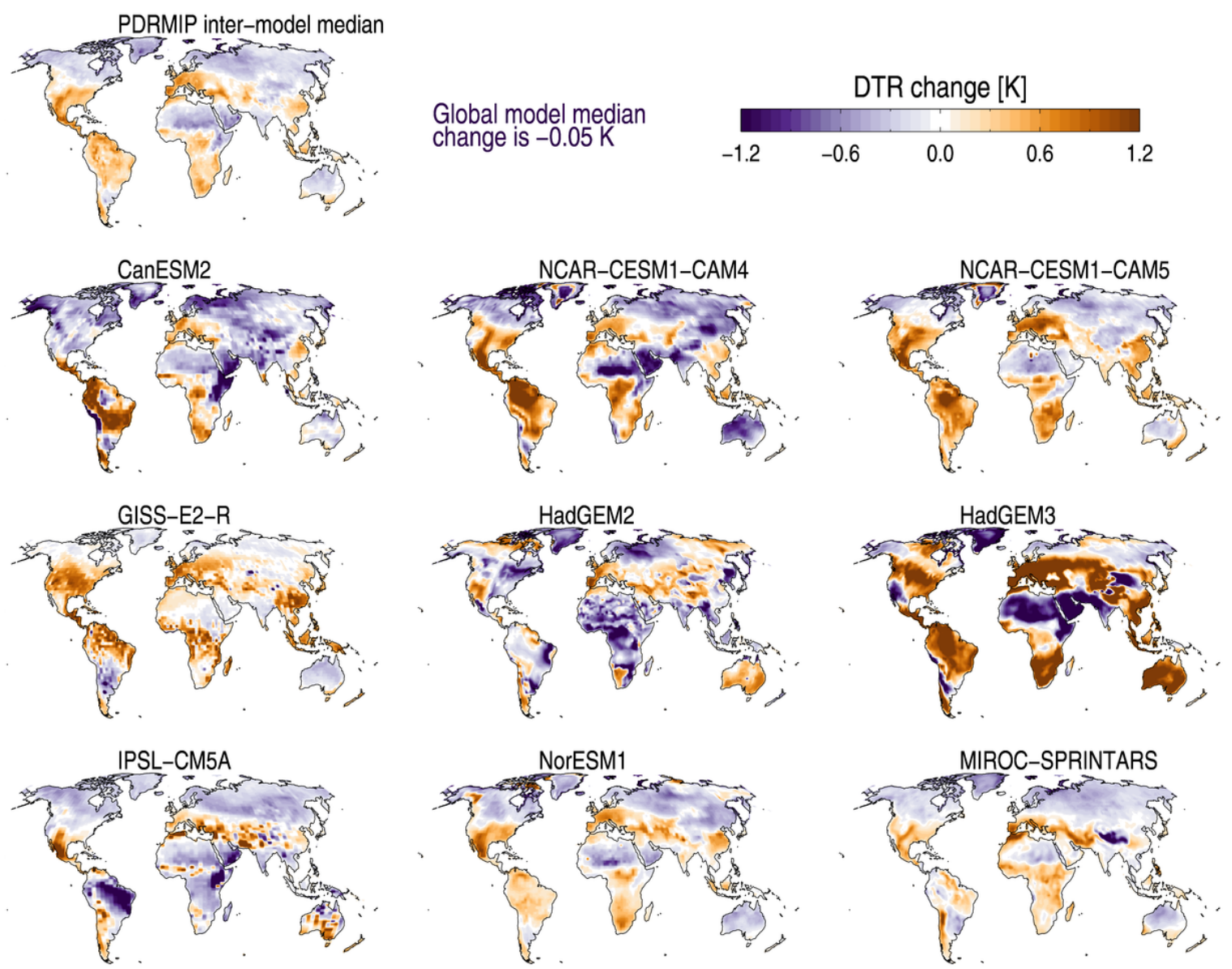

Figure S1: Annual mean DTR change (not normalized by global mean temperature change as in the main manuscript) for the $\mathrm{CO} 2 \times 2$ experiment for all nine models. 



Figure S2: Annual mean DTR change (not normalized by the global mean temperature change as in the main manuscript) for the $\mathrm{BC} \times 10$ experiment for all nine models. 

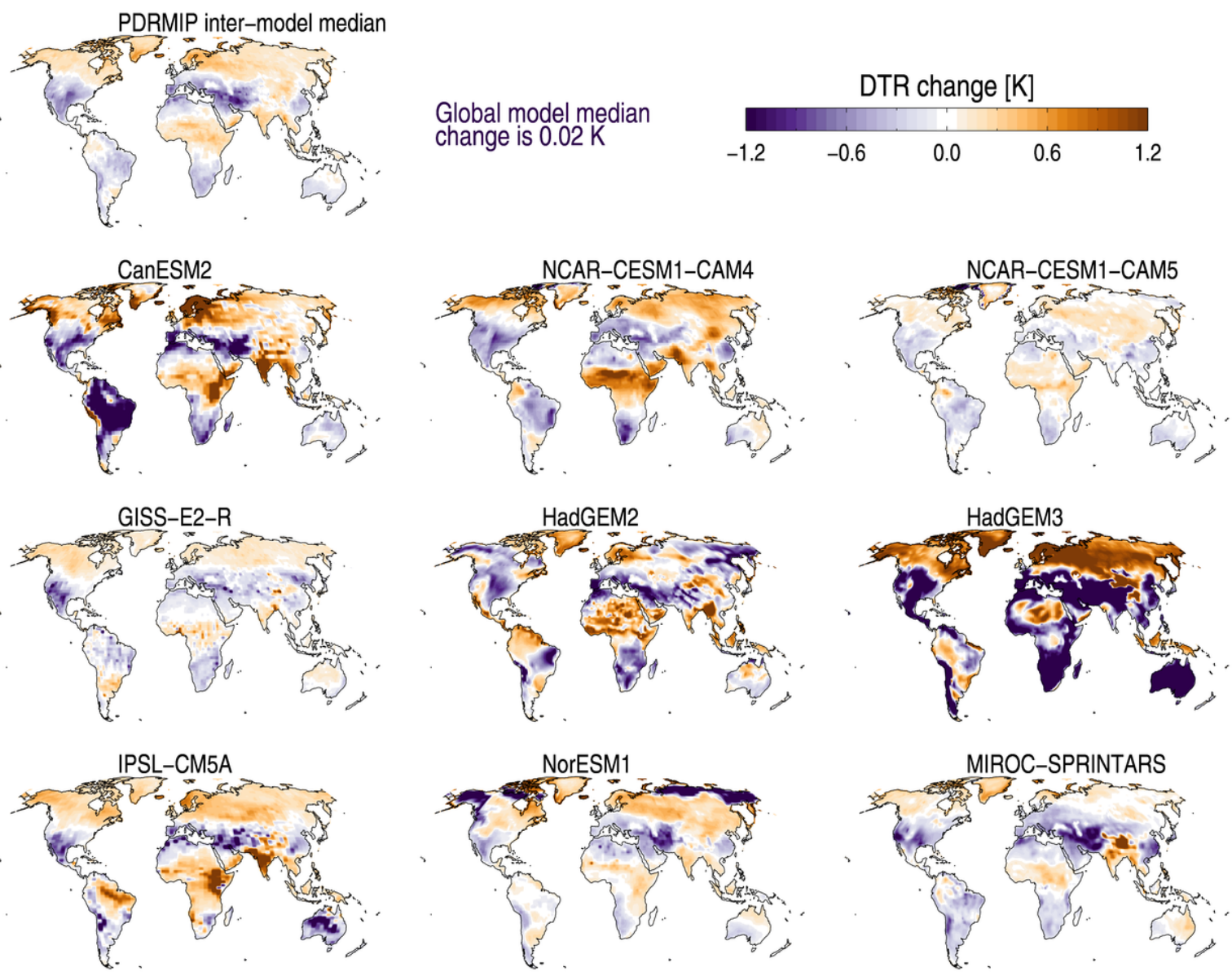

Figure S3: Annual mean DTR change (not normalized by the global mean temperature change as in the main manuscript) for the $\mathrm{SO} 4 \times 5$ experiment for all nine models. Note that as $\mathrm{SO}_{4}$ cools the climate, normalization by the global mean temperature change turns the sign of the DTR change expected from an increase in $\mathrm{SO}_{4}$. An increase in $\mathrm{SO}_{4}$ causes, e.g., reduced DTR over China and increased DTR over India, as seen in the maps above, but opposite to the signals seen in the maps of the main manuscript. 


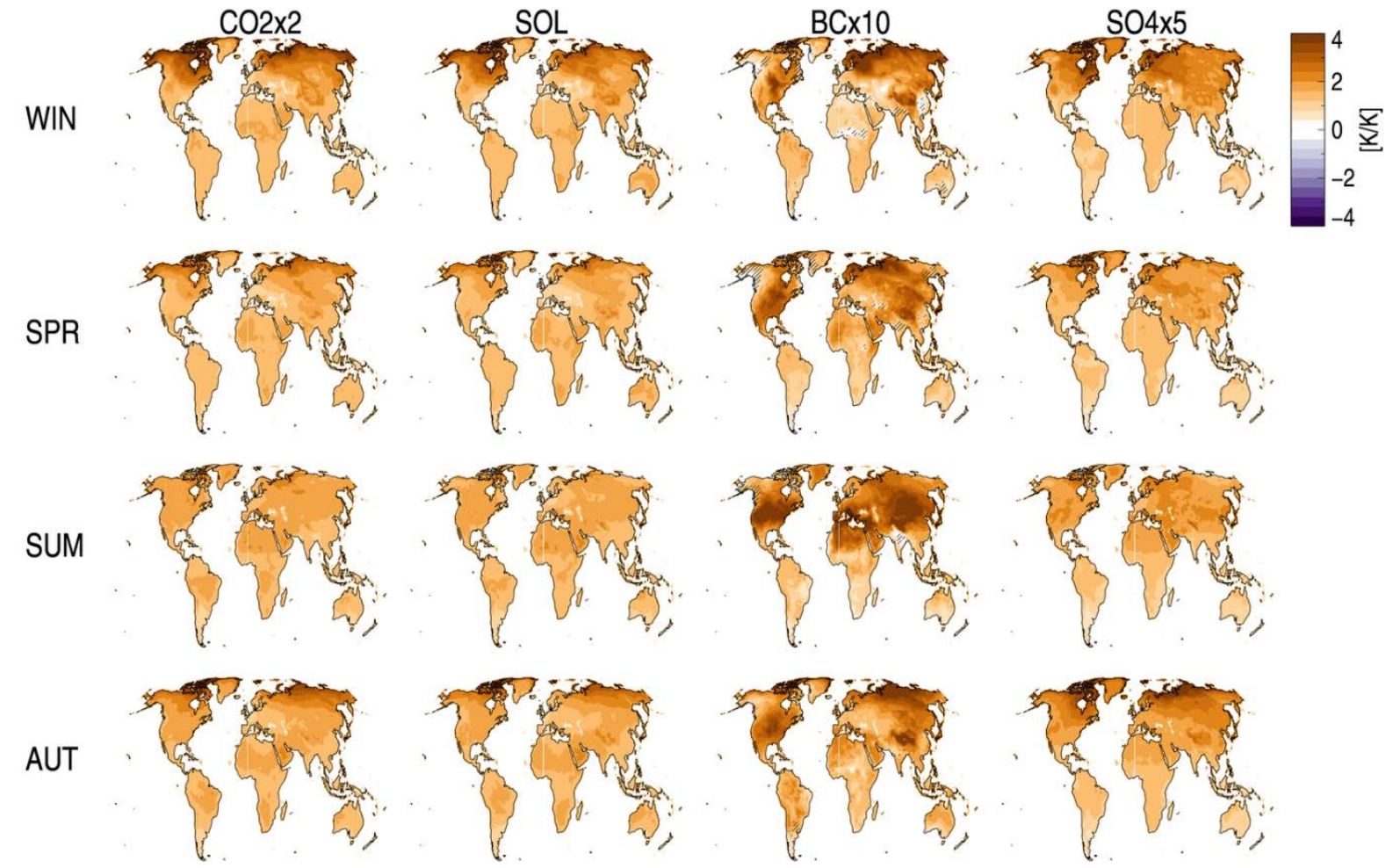

Figure S4: Multi-model median change in Tmin for the different seasons and drivers, normalized by the global mean temperature change for each model and driver. 


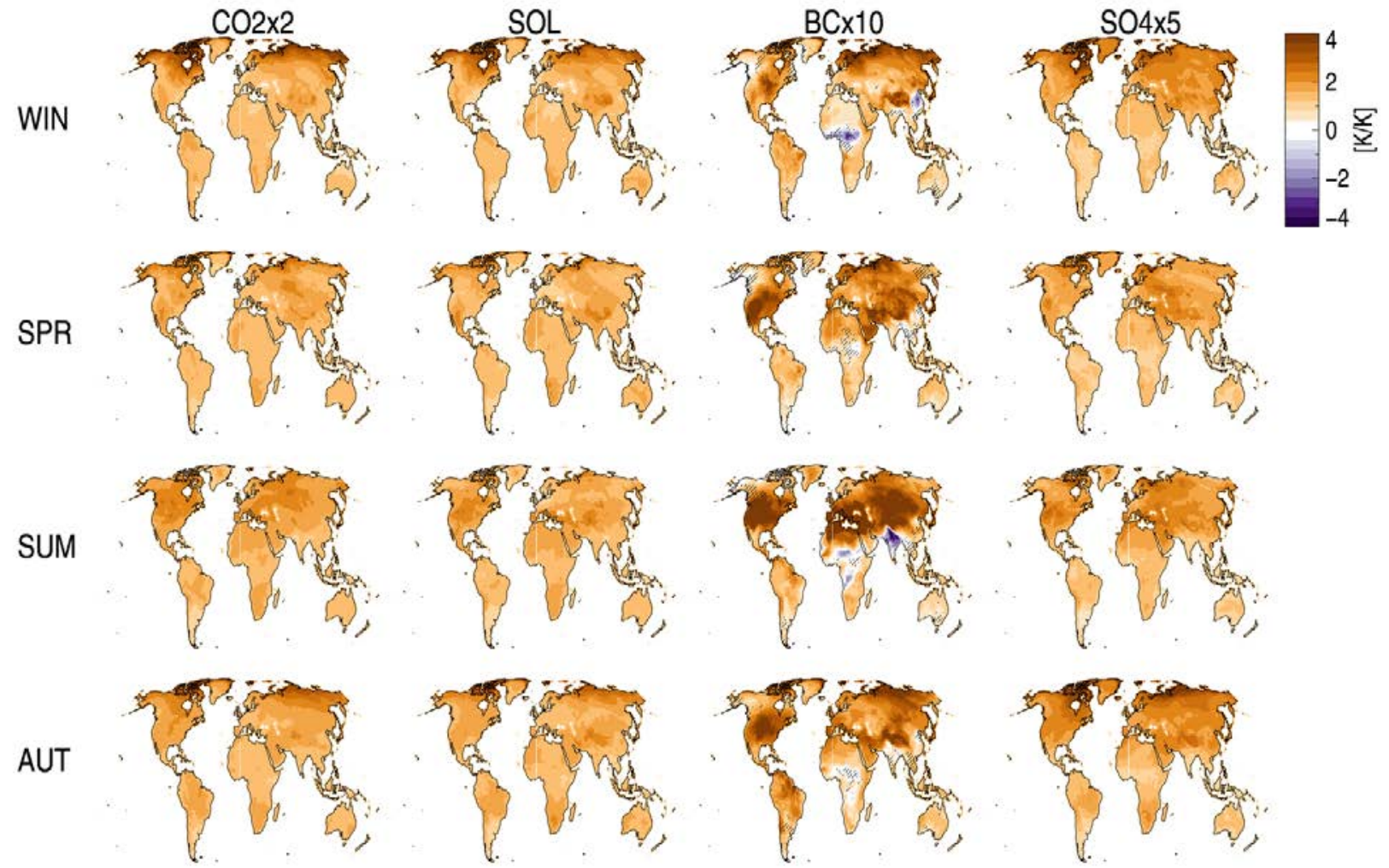

Figure S5: Multi-model median change in Tmax for the different seasons and drivers, normalized by the global mean temperature change for each model and driver. 